\title{
On the Growth of Nonoscillatory Solutions for Difference Equations with Deviating Argument
}

\author{
M. Cecchi, ${ }^{1}$ Z. Došlá, ${ }^{2}$ and M. Marini ${ }^{1}$ \\ ${ }^{1}$ Department of Electronics and Telecommunications, University of Florence, Via S. Marta 3, \\ 50139 Firenze, Italy \\ ${ }^{2}$ Department of Mathematics and Statistics, Masaryk University, Janáčkovo nám. 2a, \\ 60200 Brno, Czech Republic
}

Correspondence should be addressed to M. Cecchi, mariella.cecchi@unifi.it

Received 28 March 2008; Accepted 27 May 2008

Recommended by Martin Bohner

The half-linear difference equations with the deviating argument $\Delta\left(a_{n}\left|\Delta x_{n}\right|^{\alpha} \operatorname{sgn} \Delta x_{n}\right)+$ $b_{n}\left|x_{n+q}\right|^{\alpha} \operatorname{sgn} x_{n+q}=0, q \in \mathbb{Z}$ are considered. We study the role of the deviating argument $q$, especially as regards the growth of the nonoscillatory solutions and the oscillation. Moreover, the problem of the existence of the intermediate solutions is completely resolved for the classical half-linear equation $(q=1)$. Some analogies or discrepancies on the growth of the nonoscillatory solutions for the delayed and advanced equations are presented; and the coexistence with different types of nonoscillatory solutions is studied.

Copyright (C) $2008 \mathrm{M}$. Cecchi et al. This is an open access article distributed under the Creative Commons Attribution License, which permits unrestricted use, distribution, and reproduction in any medium, provided the original work is properly cited.

\section{Introduction}

Consider the half-linear difference equations with the deviating argument

$$
\Delta\left(a_{n}\left|\Delta x_{n}\right|^{\alpha} \operatorname{sgn} \Delta x_{n}\right)+b_{n}\left|x_{n+q}\right|^{\alpha} \operatorname{sgn} x_{n+q}=0,
$$

where $\Delta$ is the forward difference operator $\Delta x_{n}=x_{n+1}-x_{n}, \alpha>0, q \in \mathbb{Z}$ and $a=\left\{a_{n}\right\}, b=\left\{b_{n}\right\}$ are positive real sequences for $n \geq 0$ such that

$$
Y_{a}=\sum_{n=0}^{\infty}\left(\frac{1}{a_{n}}\right)^{1 / \alpha}=\infty, \quad Y_{b}=\sum_{n=0}^{\infty} b_{n}<\infty .
$$

A large number of papers deal with the oscillatory and asymptotic properties of several particular cases of (1.1), such as the classical half-linear equation

$$
\Delta\left(a_{n}\left|\Delta x_{n}\right|^{\alpha} \operatorname{sgn} \Delta x_{n}\right)+b_{n}\left|x_{n+1}\right|^{\alpha} \operatorname{sgn} x_{n+1}=0
$$


and the equations with the advanced or delayed argument $\tau \in \mathbb{Z}, \tau \geq 1$,

$$
\begin{gathered}
\Delta\left(a_{n}\left|\Delta x_{n}\right|^{\alpha} \operatorname{sgn} \Delta x_{n}\right)+b_{n}\left|x_{n+1+\tau}\right|^{\alpha} \operatorname{sgn} x_{n+1+\tau}=0, \\
\Delta\left(a_{n}\left|\Delta x_{n}\right|^{\alpha} \operatorname{sgn} \Delta x_{n}\right)+b_{n}\left|x_{n+1-\tau}\right|^{\alpha} \operatorname{sgn} x_{n+1-\tau}=0,
\end{gathered}
$$

see, for example, [1-9], the monographs [10,11], and references therein.

By solution of (1.1) we mean a nontrivial sequence satisfying (1.1) for large $n$. As usual, a solution $x=\left\{x_{n}\right\}$ of (1.1) is said to be nonoscillatory if there exists a large $n_{x}$ such that $x_{n} x_{n+1}>0$ for $n \geq n_{x}$, otherwise it is said to be oscillatory. Equation (1.1) is characterized by the homogeneity property, which means that if $x$ is a solution of (1.1), then also $\lambda x$ is its solution for any constant $\lambda$.

It is well-known that the deviating argument $\tau$ plays an important role in the oscillation. For instance, for $(\mathrm{H})$ the Sturm-type separation property holds and so all its solutions are either nonoscillatory or oscillatory, see, for example, [11, Section 8.2]. In general, this property is no true anymore for $(\mathrm{H}+)$ and $(\mathrm{H}-)$; and the coexistence of oscillatory and nonoscillatory solutions can occur even in the linear case, that is when $\alpha=1$, as we illustrate below. Nevertheless, in [4] some comparison criteria, which link the nonoscillation of $(\mathrm{H})$ with the existence of nonoscillatory solutions of the advanced equation $(\mathrm{H}+)$, or the delayed equation $(\mathrm{H}-)$, are given. In particular, if $a \equiv 1$, the nonoscillation of $(\mathrm{H})$ is equivalent to the existence of nonoscillatory solutions for $(\mathrm{H}+)$ or $(\mathrm{H}-)$, see [4, Corollary 8$]$.

Nonoscillatory solutions of (1.1) can be classified as subdominant, intermediate, or dominant solutions, according to their asymptotic behavior, see below for the definition. As it is claimed in [12, page 241], the existence of the intermediate solutions is a difficult problem also in the continuous case. Moreover, another well-known problem is their possible coexistence with different types of nonoscillatory solutions, see, for example, [13, page 213]. In [14], both problems have been completely resolved for the half-linear differential equation

$$
\left(a(t)\left|x^{\prime}\right|^{\alpha} \operatorname{sgn} x^{\prime}\right)^{\prime}+b(t)|x|^{\alpha} \operatorname{sgn} x=0,
$$

using the extension of the Wronskian. Such approach cannot be used for $(\mathrm{H})$ because the monotonicity of the corresponding Casoratian-type function remains an open problem.

The aim of this paper is to study intermediate solutions for (1.1) and the role of the deviating argument $q$, especially as regards the growth of the nonoscillatory solutions and the oscillation. The problem of the existence of intermediate solutions is completely resolved when $q=1$, that is for the half-linear equation $(\mathrm{H})$. When $q \neq 1$ some analogies or discrepancies on the growth of the nonoscillatory solutions, due to the presence of the deviating argument, are presented and also the coexistence with different types of nonoscillatory solutions is studied. Roughly speaking, if $a_{n} \equiv 1$, the deviating argument has no effect, that is (1.1) has the same types of nonoscillatory solutions for any $q$. On the other hand, if $a$ is rapidly increasing, or decreasing, for large $n$, the delay may change the type of nonoscillatory solutions as well as the oscillation, as examples below show.

\section{Main results}

For any solution $x$ of (1.1) we denote by $x^{[1]}=\left\{x_{n}^{[1]}\right\}$ its quasidifference, where

$$
x_{n}^{[1]}=a_{n}\left|\Delta x_{n}\right|^{\alpha} \operatorname{sgn} \Delta x_{n} .
$$


In view of (1.2), any nonoscillatory solution $x$ of (1.1) is eventually monotone and verifies $x_{n} x_{n}^{[1]}>0$ for large $n$; we denote this property by saying that $x$ is of class $\mathbb{M}^{+}$. Let $x$ be a solution of (1.1) in the class $\mathbb{M}^{+}$; then for large $n$ either $x$ is positive increasing and $x^{[1]}$ positive decreasing or $x$ is negative decreasing and $x^{[1]}$ negative increasing. So, we can divide the class $\mathbb{M}^{+}$into the three subclasses:

$$
\begin{aligned}
& \mathbb{M}_{\infty, \ell}^{+}=\left\{x \in \mathbb{M}^{+}: \lim _{n}\left|x_{n}\right|=\infty, \lim _{n} x_{n}^{[1]}=\ell_{x}, 0<\left|\ell_{x}\right|<\infty\right\}, \\
& \mathbb{M}_{\infty, 0}^{+}=\left\{x \in \mathbb{M}^{+}: \lim _{n}\left|x_{n}\right|=\infty, \lim _{n} x_{n}^{[1]}=0\right\}, \\
& \mathbb{M}_{\ell, 0}^{+}=\left\{x \in \mathbb{M}^{+}: \lim _{n} x_{n}=\ell_{x}, \lim _{n} x_{n}^{[1]}=0,0<\left|\ell_{x}\right|<\infty\right\},
\end{aligned}
$$

see also $[6,9]$. Following the terminology of [15] in the continuous case, solutions in $\mathbb{M}_{\infty, \ell^{\prime}}$ $\mathbb{M}_{\infty, 0}^{+}, \mathbb{M}_{\ell, 0}^{+}$are called dominant solutions, intermediate solutions, and subdominant solutions, respectively. This terminology is justified by the fact that if $x \in \mathbb{M}_{\infty, \ell}^{+}, y \in \mathbb{M}_{\infty, 0}^{+}, z \in \mathbb{M}_{\infty, \ell^{\prime}}^{+}$ then $\left|x_{n}\right|>\left|y_{n}\right|>\left|z_{n}\right|$ for large $n$.

The series

$$
\begin{gathered}
S_{\alpha}=\sum_{n=0}^{\infty}\left(\frac{1}{a_{n}}\right)^{1 / \alpha}\left(\sum_{k=n}^{\infty} b_{k}\right)^{1 / \alpha}, \\
T_{\alpha}(q)=\sum_{n=0}^{\infty} b_{n}\left(\sum_{k=0}^{n+q-1}\left(\frac{1}{a_{k}}\right)^{1 / \alpha}\right)^{\alpha},
\end{gathered}
$$

where the convention $\sum_{n_{1}}^{n_{2}} u_{i}=0$ if $n_{1}>n_{2}$ is used, play an important role in the classification of nonoscillatory solutions of (1.1). The possible cases for the behavior of these series are the following

$$
\begin{array}{ll}
\left(C_{1}\right) S_{\alpha}<\infty, & T_{\alpha}(q)<\infty ; \\
\left(C_{2}\right) S_{\alpha}=\infty, & T_{\alpha}(q)<\infty ; \\
\left(C_{3}\right) S_{\alpha}<\infty, & T_{\alpha}(q)=\infty ; \\
\left(C_{4}\right) S_{\alpha}=\infty, & T_{\alpha}(q)=\infty .
\end{array}
$$

Observe that, when $q=1$, the case $\left(C_{2}\right)$ is possible only when $\alpha>1$, while the case $\left(C_{3}\right)$ is possible only when $\alpha<1$ [2, Theorem 4]. When $q \neq 1$, in view of the fact $T_{\alpha}(1) \leq T_{\alpha}(q)$ for $q>1$, the case $\left(C_{2}\right)$ is not possible when $\alpha<1, q>1$ and the case $\left(C_{3}\right)$ is not possible when $\alpha>1$, $q<1$.

If $q<1[q>1]$, it is easy to give an example of (1.1) satisfying the case $\left(C_{2}\right)$ [the case $\left(C_{3}\right)$ ] for any $\alpha$, see Example 5.3 below.

The main results of this paper are the following.

Theorem 2.1. For $(\mathrm{H})$ we have:

(i $\left.i_{1}\right)$ if $S_{\alpha}<\infty, T_{\alpha}(1)<\infty$, then $\mathbb{M}_{\ell, 0}^{+} \neq \varnothing, \mathbb{M}_{\infty, 0}^{+}=\varnothing, \mathbb{M}_{\infty, \ell}^{+} \neq \varnothing$;

$\left(i_{2}\right)$ if $S_{\alpha}=\infty, T_{\alpha}(1)<\infty$, then $\mathbb{M}_{\ell, 0}^{+}=\varnothing, \mathbb{M}_{\infty, 0}^{+} \neq \varnothing, \mathbb{M}_{\infty, \ell}^{+} \neq \varnothing$; 
(i i ) if $S_{\alpha}<\infty, T_{\alpha}(1)=\infty$, then $\mathbb{M}_{\ell, 0}^{+} \neq \varnothing, \mathbb{M}_{\infty, 0}^{+} \neq \varnothing, \mathbb{M}_{\infty, \ell}^{+}=\varnothing$;

$\left(i_{4}\right)$ if $S_{\alpha}=\infty, T_{\alpha}(1)=\infty$ and $(\mathrm{H})$ is nonoscillatory, then $\mathbb{M}_{\ell, 0}^{+}=\varnothing, \mathbb{M}_{\infty, 0}^{+} \neq \varnothing, \mathbb{M}_{\infty, \ell}^{+}=\varnothing$.

Theorem 2.2. For (1.1) with $q \neq 1$ we have:

$\left(i_{1}\right)$ if $S_{\alpha}<\infty, T_{\alpha}(q)<\infty$, then $\mathbb{M}_{\ell, 0}^{+} \neq \varnothing, \mathbb{M}_{\infty, 0}^{+}=\varnothing, \mathbb{M}_{\infty, \ell}^{+} \neq \varnothing$;

(i $\left.i_{2}\right)$ if $S_{\alpha}=\infty, T_{\alpha}(q)<\infty$, then $\mathbb{M}_{\ell, 0}^{+}=\varnothing, \mathbb{M}_{\infty, 0}^{+} \neq \varnothing, \mathbb{M}_{\infty, \ell}^{+} \neq \varnothing$;

$\left(i_{3}\right)$ if $S_{\alpha}<\infty, T_{\alpha}(q)=\infty$ and

$$
\liminf _{n} a_{n}>0,
$$

then $\mathbb{M}_{\ell, 0}^{+} \neq \varnothing, \mathbb{M}_{\infty, 0}^{+} \neq \varnothing, \mathbb{M}_{\infty, \ell}^{+}=\varnothing ;$

$\left(i_{4}\right)$ if $S_{\alpha}=\infty, T_{\alpha}(q)=\infty$, the condition (2.8) is verified and $(\mathrm{H})$ is nonoscillatory, then $\mathbb{M}_{\ell, 0}^{+}=\varnothing$, $\mathbb{M}_{\infty, 0}^{+} \neq \varnothing, \mathbb{M}_{\infty, \ell}^{+}=\varnothing$.

Theorems 2.1, 2.2 will be proved in the following sections. Observe that Theorem 2.1 is a discrete counterpart of [14, Theorems 4, 6, 7] for (1.3), even if the approach here used is completely different.

\section{Unbounded solutions when $T_{\alpha}(q)<\infty$}

In this section we study the growth of unbounded solutions of $(1.1)$ when $T_{\alpha}(q)<\infty$. The following holds.

Theorem 3.1. Assume

$$
S_{\alpha}<\infty, \quad T_{\alpha}(q)<\infty
$$

Then for (1.1) we have $\mathbb{M}_{\infty, 0}^{+}=\varnothing$.

Proof. By contradiction, assume that $x \in \mathbb{M}_{\infty, 0}^{+}$. Without loss of generality, let $n_{0}$ be large so that $n_{0}+\min \{q, 0\} \geq 0$ and

$$
x_{n}>0, \quad x_{n+q}>0, \quad \Delta x_{n}>0, \quad \Delta x_{n+q}>0 \quad \text { for } n \geq n_{0} .
$$

Set $n_{q}=n_{0}+q$; then $x_{n+q}>x_{n_{q}}$ for $n>n_{0}$. By summation of (1.1) we obtain, for $n>n_{0}$,

$$
x_{n}^{[1]}=\sum_{k=n}^{\infty} b_{k}\left(x_{k+q}\right)^{\alpha}
$$

and so

$$
x_{n+q}-x_{n_{q}}=\sum_{k=n_{q}}^{n+q-1}\left(\frac{1}{a_{k}}\right)^{1 / \alpha}\left(\sum_{i=k}^{n} b_{i}\left(x_{i+q}\right)^{\alpha}+\sum_{i=n+1}^{\infty} b_{i}\left(x_{i+q}\right)^{\alpha}\right)^{1 / \alpha} .
$$

Putting

$$
\sigma_{\alpha}= \begin{cases}1, & \text { if } \alpha \geq 1 \\ 2^{(1-\alpha) / \alpha}, & \text { if } \alpha<1\end{cases}
$$


and using (3.3) and the inequality

$$
(X+Y)^{1 / \alpha} \leq \sigma_{\alpha}\left(X^{1 / \alpha}+Y^{1 / \alpha}\right)
$$

we obtain the following, for $n>n_{0}$ :

$$
\begin{aligned}
x_{n+q}-x_{n_{q}} & \leq \sigma_{\alpha} \sum_{k=n_{q}}^{n+q-1}\left(\frac{1}{a_{k}}\right)^{1 / \alpha}\left(\sum_{i=k}^{n} b_{i}\left(x_{i+q}\right)^{\alpha}\right)^{1 / \alpha}+\sigma_{\alpha} \sum_{k=n_{q}}^{n+q-1}\left(\frac{1}{a_{k}}\right)^{1 / \alpha}\left(\sum_{i=n+1}^{\infty} b_{i}\left(x_{i+q}\right)^{\alpha}\right)^{1 / \alpha} \\
& \leq \sigma_{\alpha} x_{n+q} \sum_{k=n_{q}}^{n+q-1}\left(\frac{1}{a_{k}}\right)^{1 / \alpha}\left(\sum_{i=k}^{n} b_{i}\right)^{1 / \alpha}+\sigma_{\alpha}\left(x_{n+1}^{[1]}\right)^{1 / \alpha} \sum_{k=n_{q}}^{n+q-1}\left(\frac{1}{a_{k}}\right)^{1 / \alpha}
\end{aligned}
$$

and so

$$
x_{n+q}-x_{n_{q}} \leq \sigma_{\alpha} x_{n+q} \sum_{k=n_{q}}^{\infty}\left(\frac{1}{a_{k}}\right)^{1 / \alpha}\left(\sum_{i=k}^{\infty} b_{i}\right)^{1 / \alpha}+\sigma_{\alpha}\left(x_{n+1}^{[1]}\right)^{1 / \alpha} \sum_{k=n_{q}}^{n+q-1}\left(\frac{1}{a_{k}}\right)^{1 / \alpha} .
$$

Therefore,

$$
1 \leq \frac{x_{n_{q}}}{x_{n+q}}+\sigma_{\alpha} \sum_{k=n_{q}}^{\infty}\left(\frac{1}{a_{k}}\right)^{1 / \alpha}\left(\sum_{i=k}^{\infty} b_{i}\right)^{1 / \alpha}+\frac{\sigma_{\alpha}\left(x_{n+1}^{[1]}\right)^{1 / \alpha}}{x_{n+q}} \sum_{k=n_{q}}^{n+q-1}\left(\frac{1}{a_{k}}\right)^{1 / \alpha}
$$

In view of $S_{\alpha}<\infty$, fixed $\varepsilon, 0<\varepsilon<1$, we can choose $n_{q}$ large so that

$$
\sigma_{\alpha} \sum_{k=n_{q}}^{\infty}\left(\frac{1}{a_{k}}\right)^{1 / \alpha}\left(\sum_{i=k}^{\infty} b_{i}\right)^{1 / \alpha}<\varepsilon / 2
$$

Since $x$ is unbounded, there exists $N>n_{0}$ such that for $n \geq N$,

$$
\frac{x_{n_{q}}}{x_{n+q}}<\varepsilon / 2
$$

Hence, from (3.9) we obtain that there exists $\gamma>0$ such that for $n \geq N$,

$$
\left(x_{n+1}^{[1]}\right)^{1 / \alpha} \sum_{k=n_{q}}^{n+q-1}\left(\frac{1}{a_{k}}\right)^{1 / \alpha} \geq \gamma x_{n+q}
$$

where $\gamma=(1-\varepsilon) / \sigma_{\alpha}$. Summing (1.1) we obtain

$$
x_{N}^{[1]}-x_{n+1}^{[1]}=\sum_{k=N}^{n} b_{k}\left(x_{k+q}\right)^{\alpha},
$$


that is, in view of (3.12),

$$
\gamma^{\alpha}\left(x_{N}^{[1]}-x_{n+1}^{[1]}\right) \leq \sum_{k=N}^{n} b_{k} x_{k+1}^{[1]}\left(\sum_{i=n_{q}}^{k+q-1}\left(\frac{1}{a_{i}}\right)^{1 / \alpha}\right)^{\alpha} .
$$

Since $x^{[1]}$ is decreasing, we have

$$
\gamma^{\alpha}\left(x_{N}^{[1]}-x_{n+1}^{[1]}\right) \leq x_{N}^{[1]} \sum_{k=N}^{\infty} b_{k}\left(\sum_{i=n_{q}}^{k+q-1}\left(\frac{1}{a_{i}}\right)^{1 / \alpha}\right)^{\alpha} .
$$

In view of $T_{\alpha}(q)<\infty$, let $N$ be large so that

$$
\sum_{k=N}^{\infty} b_{k}\left(\sum_{i=n_{q}}^{k+q-1}\left(\frac{1}{a_{i}}\right)^{1 / \alpha}\right)^{\alpha} \leq \gamma^{\alpha} / 2
$$

Thus, from (3.15) we obtain $\left(\gamma^{\alpha} / 2\right) x_{N}^{[1]} \leq \gamma^{\alpha} x_{n+1}^{[1]}$, which is a contradiction because $x^{[1]}$ tends to zero as $n \rightarrow \infty$.

The following result extends [3, Theorem 2], where the existence of intermediate solutions has been proved for $q \geq 0$.

Theorem 3.2. ( $\left.i_{1}\right)$ Equation (1.1) has solutions in the class $\mathbb{M}_{\infty, \ell}^{+}$if and only if $T_{\alpha}(q)<\infty$.

$\left(i_{2}\right)$ Equation (1.1) has solutions in $\mathbb{M}_{\infty, 0}^{+}$if $S_{\alpha}=\infty$ and $T_{\alpha}(q)<\infty$.

Proof (outline). If $q \geq 0$, claim ( $\left.i_{1}\right)$ follows, for example, from [6, Theorem 3 ] with minor changes and claim $\left(i_{2}\right)$ from [3, Theorem 2]. Now we sketch the existence part of claims $\left(i_{1}\right),\left(i_{2}\right)$ when $q<0$.

Assume $T_{\alpha}(q)<\infty$ and let $\delta \in\{0,1\}$. Using the comparison criterion, the series

$$
\sum_{k=1}^{\infty} b_{k}\left(\delta+\sum_{i=1}^{k+q-1} \frac{1}{\left(a_{i}\right)^{1 / \alpha}}\right)^{\alpha}
$$

converges, too. So, choose $n_{0}$ large so that $n_{0}+q \geq 2$ and

$$
\sum_{k=n_{0}}^{\infty} b_{k}\left(\delta+\sum_{i=1}^{k+q-1} \frac{1}{\left(a_{i}\right)^{1 / \alpha}}\right)^{\alpha}<1, \quad \sum_{j=1}^{n_{0}+q-1} \frac{1}{\left(a_{j}\right)^{1 / \alpha}} \geq 1
$$

Set $n_{q}=n_{0}+q$ and denote by $\mathbb{X}$ the Fréchet space of the real sequences defined for $n \geq n_{q}$, endowed with the topology of convergence on finite subsets of $\mathbb{N}_{n_{q}}=\left\{n \in \mathbb{N}, n \geq n_{q}\right\}$. Consider the subset

$$
\Omega=\left\{u=\left\{u_{n}\right\} \in \mathbb{X}: 1 \leq u_{n} \leq \sum_{j=1}^{n-1} \frac{1}{\left(a_{j}\right)^{1 / \alpha}}\right\}
$$


and define the operator $\tau: \Omega \rightarrow \mathbb{X}$ given by $\tau(u)=y$, where

$$
\begin{aligned}
& y_{n}=1 \quad \text { if } n_{q} \leq n \leq n_{0} \\
& y_{n}=1+\sum_{j=n_{0}}^{n-1} \frac{1}{\left(a_{j}\right)^{1 / \alpha}}\left(\delta+\sum_{i=j}^{\infty} b_{i}\left(u_{i+q}\right)^{\alpha}\right)^{1 / \alpha}, \quad \text { if } n>n_{0} .
\end{aligned}
$$

For $i \geq n_{0}$ it results $i+q \geq n_{q}$ and so we have for $u \in \Omega$

$$
u_{i+q} \leq \sum_{k=1}^{i+q-1} \frac{1}{\left(a_{k}\right)^{1 / \alpha}} .
$$

Then, in view of (3.18) we obtain the following for $n>n_{0}$ :

$$
\begin{aligned}
y_{n} & \leq 1+\sum_{j=n_{0}}^{n-1} \frac{1}{\left(a_{j}\right)^{1 / \alpha}}\left(\sum_{i=j}^{\infty} b_{i}\left(\delta+\sum_{k=1}^{i+q-1} \frac{1}{\left(a_{k}\right)^{1 / \alpha}}\right)^{\alpha}\right)^{1 / \alpha} \\
& \leq 1+\left(\sum_{j=n_{0}}^{n-1} \frac{1}{\left(a_{j}\right)^{1 / \alpha}}\right)\left(\sum_{i=n_{0}}^{\infty} b_{i}\left(\delta+\sum_{k=1}^{i+q-1} \frac{1}{\left(a_{k}\right)^{1 / \alpha}}\right)^{\alpha}\right)^{1 / \alpha} \\
& \leq 1+\sum_{j=n_{0}}^{n-1} \frac{1}{\left(a_{j}\right)^{1 / \alpha}} \leq \sum_{j=1}^{n-1} \frac{1}{\left(a_{j}\right)^{1 / \alpha}} .
\end{aligned}
$$

If $n_{q} \leq n \leq n_{0}$, from (3.18) we have

$$
y_{n}=1 \leq \sum_{j=1}^{n_{q}-1} \frac{1}{\left(a_{j}\right)^{1 / \alpha}} \leq \sum_{j=1}^{n-1} \frac{1}{\left(a_{j}\right)^{1 / \alpha}}
$$

and so $\tau$ maps $\Omega$ into itself. In virtue of the Ascoli theorem, any bounded set in $\mathbb{X}$ is relatively compact and so, because $\tau(\Omega)$ is bounded according to the topology of $\mathbb{X}$, the compactness follows. Let $\left\{v^{(k)}\right\}$ be a sequence in $\Omega$, converging on finite subsets of $\mathbb{N}_{n_{q}}$ to $v^{(\infty)} \in \Omega$. Using the discrete analogue of the Lebesgue dominated convergence theorem, the sequence $\left\{\tau\left(v^{(k)}\right)\right\}$ converges on finite subsets of $\mathbb{N}_{n_{q}}$ to $\tau\left(v^{(\infty)}\right)$; and so the continuity of $\tau$ is proved. So, by applying the Tychonov fixed point theorem, there exists a solution $x$ of (1.1) which satisfies for large $n$

$$
\begin{gathered}
x_{n} \geq 1+\sum_{j=n_{0}}^{n-1} \frac{1}{\left(a_{j}\right)^{1 / \alpha}}\left(\delta+\sum_{i=j}^{\infty} b_{i}\right)^{1 / \alpha}, \\
\delta+\sum_{i=n}^{\infty} b_{i} \leq x_{n}^{[1]} \leq \delta+\sum_{i=n}^{\infty} b_{i}\left(\sum_{k=1}^{i+q-1} \frac{1}{\left(a_{k}\right)^{1 / \alpha}}\right)^{\alpha} .
\end{gathered}
$$

If $\delta=1$, then $\lim _{n} x_{n}^{[1]}=1$ and so $x \in \mathbb{M}_{\infty, \ell}^{+}$. If $\delta=0$ and $S_{\alpha}=\infty$, then $x \in \mathbb{M}_{\infty, 0}^{+}$and the proof is complete. 


\section{Bounded solutions and proof of Theorem 2.1}

It is well-known that the existence of bounded solutions of (1.1) depends on the convergence of the series $S_{\alpha}$, and so the deviating argument does not play any role. This property follows, for example, from [9, Theorem 4.2] or [6, Theorem 2], in which the case $q \geq 0$ is considered, but the used argument can be easily modified for any $q \in \mathbb{Z}$. More precisely the following holds.

Proposition 4.1. Equation (1.1) has solutions in the class $\mathbb{M}_{\ell, 0}^{+}$if and only if $S_{\alpha}<\infty$.

When $q=1$, bounded solutions of $(\mathrm{H})$ are uniquely determined up to a constant factor. To prove this, we use a uniqueness result, stated in [1, Theorem 1], and the so-called reciprocity principle (see, e.g., [11, Section 8.2.2]), which links solutions of $(\mathrm{H})$ with ones of the reciprocal equation $(n \geq 1)$ :

$$
\Delta\left(B_{n}\left|\Delta y_{n}\right|^{1 / \alpha} \operatorname{sgn} \Delta y_{n}\right)+A_{n}\left|y_{n+1}\right|^{1 / \alpha} \operatorname{sgn} y_{n+1}=0
$$

where

$$
B_{n}=\frac{1}{\left(b_{n-1}\right)^{1 / \alpha}}, \quad A_{n}=\frac{1}{\left(a_{n}\right)^{1 / \alpha}} .
$$

Indeed, let $y$ be a solution of (4.1) and denote by $y^{[1]}$ its quasidifference, that is $y_{n}^{[1]}=$ $B_{n}\left|\Delta y_{n}\right|^{1 / \alpha} \operatorname{sgn} \Delta y_{n}$. Then the sequence $x$, where $x_{n}=-y_{n}^{[1]}$, is a solution of $(\mathrm{H})$ and satisfies

$$
x_{n}^{[1]}=y_{n}, \quad \operatorname{sgn} y_{n} y_{n}^{[1]}=-\operatorname{sgn} x_{n} x_{n}^{[1]} .
$$

Proposition 4.2. If $Y_{a}=\infty$ and $S_{\alpha}<\infty$, then for any fixed $c \neq 0,(\mathrm{H})$ has a unique solution $x \in \mathbb{M}_{\ell, 0}^{+}$ such that $\lim _{n} x_{n}=c$.

Proof. First, observe that $Y_{b}<\infty$, because $S_{\alpha}<\infty$. Consider (4.1), then

$$
S_{\alpha}=\sum_{n=0}^{\infty}\left(\frac{1}{a_{n}}\right)^{1 / \alpha}\left(\sum_{k=n+1}^{\infty} b_{k-1}\right)^{1 / \alpha}=\sum_{n=0}^{\infty} A_{n}\left(\sum_{k=n+1}^{\infty}\left(\frac{1}{B_{k}}\right)^{\alpha}\right)^{1 / \alpha}<\infty .
$$

By applying [1, Theorem 1] to (4.1), there exists a unique solution $y$ of $(4.1)$ such that $\lim _{n} y_{n}=$ $0, \lim _{n} y_{n}^{[1]}=-c$. Applying the reciprocity principle, $(\mathrm{H})$ has a unique solution $x$ such that $\lim _{n} x_{n}=c$ and $\lim _{n} x_{n}^{[1]}=0$. Since (1.2) holds, $x \in \mathbb{M}_{\ell, 0}^{+}$.

Using the above results, we can prove our first main result.

Proof of Theorem 2.1. The claims $\left(i_{k}\right), k=1,2,4$, follow from Theorems 3.1, 3.2 and Proposition 4.1.

Claim $\left(i_{3}\right)$. Let $x$ be the unique solution of $(\mathrm{H})$, defined for $n \geq N$, satisfying $x \in \mathbb{M}_{\ell, 0}^{+}$and $\lim _{n} x_{n}=1$. Let $y$ be a solution of $(\mathrm{H})$ such that $y_{N}=x_{N}$ and $y_{N+1} \not \equiv x_{N+1}$. If $y \in \mathbb{M}_{\ell, 0}^{+}$, we have

$$
\lim _{n} y_{n}=y_{\infty} \neq 1, \quad 0<\left|y_{\infty}\right|<\infty
$$


In view of the homogeneity property, also $z=\left(y_{\infty}\right)^{-1} y$ is a solution of $(\mathrm{H})$, and $\lim _{n} z_{n}=1$, $\lim _{n} z_{n}^{[1]}=0$. Then, from Proposition 4.2, we have $z \equiv x$, that is $y \equiv y_{\infty} x$. Hence $y_{N}=y_{\infty} x_{N}$ and so $y_{\infty}=1$, which contradicts (4.5). Thus $y \notin \mathbb{M}_{\ell, 0}^{+}$. Since $T_{\alpha}(1)=\infty$ and all solutions of $(\mathrm{H})$ are nonoscillatory, from Theorem 3.2 the assertion follows.

In the proof of Theorem 2.1 we used the Sturm separation property saying that oscillatory and nonoscillatory solutions cannot coexist for $(\mathrm{H})$. Nevertheless, when $q \neq 1$, such a property can fail, as the following example shows.

Example 4.3. Consider the difference equations

$$
\begin{aligned}
& \Delta^{2} x_{n}+b_{n} x_{n+1}=0 \\
& \Delta^{2} y_{n}+b_{n} y_{n+3}=0
\end{aligned}
$$

where

$$
b_{n}=2^{-6 n-12}\left(2^{4 n+6}+2^{2 n+3}+1\right) .
$$

Since $S_{1}<\infty$, by Proposition 4.1 both equations have bounded nonoscillatory solutions. Thus, because the Sturm separation property holds for (4.6), this equation has all solutions nonoscillatory. However (4.7) has the oscillatory solution $y=\left\{(-1)^{n} 2^{n(n+1)}\right\}$. Similarly,

$$
\Delta^{2} z_{n}+B_{n} z_{n-5}=0, \quad B_{n}=3^{-10 n+25}\left(3^{-4 n-4}+2 \cdot 3^{-2 n-1}+1\right)
$$

has the oscillatory solution $z=\left\{(-1)^{n} 3^{-n^{2}}\right\}$ and, again in view of Proposition 4.1, a bounded nonoscillatory solution, while the corresponding linear equation $\Delta^{2} x_{n}+B_{n} x_{n+1}=0$ is nonoscillatory, as it follows from Proposition 4.1 and the Sturm separation property.

As far as we know in the literature criteria assuring that all solutions of (1.1) with $q \neq 1$ are nonoscillatory are not available. This fact yields a strong difficulty to prove the existence of intermediate solutions of (1.1) when $T_{\alpha}(q)=\infty, q \neq 1$. These difficulties can be overcome by making a comparison result for intermediate solutions of (1.1) with $q \neq 1$ and $(\mathrm{H})$, as it is described in the following section.

\section{Comparison result and proof of Theorem 2.2}

Clearly, the convergence of the series $T_{\alpha}(q)$ depends on the deviating argument $q$. The following example illustrates this fact and shows how the presence of the deviating argument can modify the growth of nonoscillatory solutions.

Example 5.1. Let $0<\alpha<1$ and define the sequences $a, b$ so that

$$
\sum_{i=0}^{k}\left(\frac{1}{a_{i}}\right)^{1 / \alpha}=2^{k^{2}}, \quad b_{k}=2^{-\alpha k^{2}} \frac{1}{k+1} .
$$

Hence

$$
T_{\alpha}(0)=\sum_{k=1}^{\infty} b_{k}\left(\sum_{i=0}^{k-1}\left(\frac{1}{a_{i}}\right)^{1 / \alpha}\right)^{\alpha}=\sum_{k=1}^{\infty} \frac{1}{k+1} 2^{-\alpha(2 k-1)}<\infty,
$$


while

$$
T_{\alpha}(1)=\sum_{k=0}^{\infty} \frac{1}{k+1} 2^{-\alpha k^{2}} 2^{\alpha k^{2}}=\sum_{k=0}^{\infty} \frac{1}{k+1}=\infty .
$$

Moreover, if $0<X<1$, we have $(i \geq 0)$

$$
-\Delta\left(X^{i^{2}}\right)=X^{i^{2}}-X^{(i+1)^{2}}=X^{i^{2}}\left(1-X^{2 i-1}\right)>(1-X) X^{i^{2}},
$$

and so, taking $X=2^{-\alpha}$, we obtain

$$
\sum_{i=k}^{\infty} 2^{-\alpha i^{2}}<-\beta \sum_{i=k}^{\infty} \Delta\left(2^{-\alpha i^{2}}\right)=\beta 2^{-\alpha k^{2}},
$$

where $\beta=\left(1-2^{-\alpha}\right)^{-1}$. Hence

$$
\begin{aligned}
S_{\alpha} & =\sum_{k=0}^{\infty}\left(\frac{1}{a_{k}}\right)^{1 / \alpha}\left(\sum_{i=k}^{\infty} b_{i}\right)^{1 / \alpha} \\
& \leq \sum_{k=0}^{\infty} 2^{k^{2}}\left(\frac{1}{k+1}\right)^{1 / \alpha}\left(\sum_{i=k}^{\infty} 2^{-\alpha i^{2}}\right)^{1 / \alpha} \\
& \leq \beta^{1 / \alpha} \sum_{k=0}^{\infty} 2^{k^{2}}\left(\frac{1}{k+1}\right)^{1 / \alpha} 2^{-k^{2}}<\infty .
\end{aligned}
$$

Taking into account that $T_{\alpha}(p) \leq T_{\alpha}(q)$ for $p \leq q$, we have

$$
S_{\alpha}<\infty, \quad T_{\alpha}(q)<\infty \text { for } q \leq 0, T_{\alpha}(1)=\infty
$$

and so the case $\left(C_{3}\right)$ and $\left(C_{1}\right)$ occurs for $(\mathrm{H})$ and $(\mathrm{H}-)$ for $\tau \geq 1$, respectively. Therefore, the delayed argument changes the growth of unbounded solutions: all unbounded solutions of $(\mathrm{H})$ are intermediate in virtue of Theorem $2.1\left(i_{3}\right)$, while all unbounded solutions of $(\mathrm{H}-)$ are dominant for any delay $\tau$ in virtue of Theorems 3.1 and 3.2.

Now we state a comparison result for intermediate solutions of (1.1).

Theorem 5.2. Assume (2.8). If (1.1) has solutions in the class $\mathbb{M}_{\infty, 0}^{+}$for a fixed $q$, then the same occurs for any $q$.

Proof. Jointly with (1.1), consider the nonlinear difference equations

$$
\begin{aligned}
& \Delta\left(a_{n}\left|\Delta y_{n}\right|^{\alpha} \operatorname{sgn} \Delta y_{n}\right)+b_{n}\left|x_{n+q-1}\right|^{\alpha} \operatorname{sgn} y_{n+q-1}=0, \\
& \Delta\left(a_{n}\left|\Delta x_{n}\right|^{\alpha} \operatorname{sgn} \Delta x_{n}\right)+b_{n}\left|x_{n+q+1}\right|^{\alpha} \operatorname{sgn} x_{n+q+1}=0 .
\end{aligned}
$$

It is sufficient to prove that if $\mathbb{M}_{\infty, 0}^{+} \neq \varnothing$ for (1.1), then the same holds for (5.9) and (5.8). Put $\bar{q}=\min \{0, q\}$. Let $z$ be a solution of (1.1) in the class $\mathbb{M}_{\infty, 0}^{+}$and let $n_{0}$ be a positive integer so that $n_{0}+\bar{q} \geq 1$. Set

$$
\bar{n}=n_{0}+\bar{q}-1, \quad \tilde{n}=\bar{n}+1=n_{0}+\bar{q} .
$$


Then

$$
0 \leq \bar{n}<\tilde{n} \leq n_{0}
$$

Without loss of generality, assume $z_{i}>0, z_{i}^{[1]}>0$ for $i \geq \bar{n}$ and

$$
a_{i}>h,
$$

where $h$ is a positive constant. Since $z^{[1]}$ is positive decreasing for $n \geq \bar{n}$, we have $z_{n}^{[1]} \leq z_{\bar{n}}^{[1]}$ for $n \geq \bar{n}$, that is,

$$
z_{n+1}-z_{n} \leq\left(\frac{z_{\bar{n}}^{[1]}}{a_{n}}\right)^{1 / \alpha} \leq\left(\frac{z_{\bar{n}}^{[1]}}{h}\right)^{1 / \alpha}=h_{1} .
$$

Summing twice (1.1), we obtain, for $n>n_{0}$,

$$
z_{n}=z_{n_{0}}+\sum_{i=n_{0}}^{n-1}\left(\frac{1}{a_{i}}\right)^{1 / \alpha}\left(\sum_{k=i}^{\infty} b_{k}\left(z_{k+q}\right)^{\alpha}\right)^{1 / \alpha}
$$

Step $1\left(\mathbb{M}_{\infty, 0}^{+} \neq \varnothing\right.$ for $(1.1) \Rightarrow \mathbb{M}_{\infty, 0}^{+} \neq \varnothing$ for (5.8)). Denote by $\mathbb{X}$ the Fréchet space of the real sequences defined for $n \geq \bar{n}$, endowed with the topology of convergence on finite subsets of $\mathbb{N}_{\bar{n}}=\{n \in \mathbb{N}, n \geq \bar{n}\}$ and consider the set $\Omega \subset \mathbb{X}$ defined by

$$
\Omega=\left\{v=\left\{v_{n}\right\} \in \mathbb{X}: z_{n+1} \leq v_{n} \leq M z_{n+1}\right\},
$$

where

$$
M=1+\frac{h_{1}}{z_{n_{0}}}
$$

Let $\tau: \Omega \rightarrow \mathbb{X}$ be the map given by

$$
\begin{aligned}
& \tau(v)_{n}=z_{n+1} \quad \text { if } \bar{n} \leq n \leq n_{0}, \\
& \tau(v)_{n}=d+\sum_{i=n_{0}}^{n-1}\left(\frac{1}{a_{i}}\right)^{1 / \alpha}\left(\sum_{k=i}^{\infty} b_{k}\left(v_{k+q-1}\right)^{\alpha}\right)^{1 / \alpha} \quad \text { if } n \geq n_{0}+1,
\end{aligned}
$$

where

$$
d=z_{n_{0}}+h_{1}
$$

Let $\bar{n} \leq n \leq n_{0}$; clearly

$$
z_{n+1}=\tau(v)_{n}<M z_{n+1}
$$

Now let $n>n_{0}$. Since $n+q-1 \geq n_{0}+\bar{q}-1=\bar{n}$, we have for $v \in \Omega$ and $k \geq n_{0}$

$$
z_{k+q} \leq v_{k+q-1} \leq M z_{k+q}
$$


Then, from (5.14) and (5.20), we get

$$
\tau(v)_{n} \leq d+\sum_{i=n_{0}}^{n-1}\left(\frac{1}{a_{i}}\right)^{1 / \alpha}\left(\sum_{k=i}^{\infty} b_{k}\left(M z_{k+q}\right)^{\alpha}\right)^{1 / \alpha}=M z_{n}-M z_{n_{0}}+d
$$

Since from (5.16) it results $M z_{n_{0}}=z_{n_{0}}+h_{1}$, from (5.21) we obtain

$$
\tau(v)_{n} \leq M z_{n}-z_{n_{0}}-h_{1}+d=M z_{n}<M z_{n+1} .
$$

Moreover, using again (5.14) and (5.20), we obtain, for $n>n_{0}$,

$$
\tau(v)_{n} \geq d+\sum_{i=n_{0}}^{n-1}\left(\frac{1}{a_{i}}\right)^{1 / \alpha}\left(\sum_{k=i}^{\infty} b_{k}\left(z_{k+q}\right)^{\alpha}\right)^{1 / \alpha}=z_{n}-z_{n_{0}}+d=z_{n}+h_{1}
$$

that is, in view of (5.13),

$$
\tau(v)_{n} \geq z_{n+1}
$$

Hence, from (5.19), (5.22), and (5.24) we have $\tau(\Omega) \subset \Omega$. Reasoning as in the proof of Theorem 3.2, the continuity and compactness of $\tau$ in $\Omega$ follows and so, by applying the Tychonov fixed point theorem, there exists $y$ such that $y=\tau(y)$. It is easy to verify that $y$ is a solution of (5.8) for large $n$ and, since $y \in \Omega$, we have $y \in \mathbb{M}_{\infty, 0}^{+}$, that is, the assertion.

Step $2\left(\mathbb{M}_{\infty, 0}^{+} \neq \varnothing\right.$ for $(1.1) \Rightarrow \mathbb{M}_{\infty, 0}^{+} \neq \varnothing$ for (5.9)). Denote by $\mathbb{X}$ the Fréchet space of the real sequences defined for $n \geq \tilde{n}$, endowed with the topology of convergence on finite subsets of $\mathbb{N}_{\tilde{n}}=\{n \in \mathbb{N}, n \geq \tilde{n}\}$, and consider the set $\Omega \subset \mathbb{X}$ defined by

$$
\Omega=\left\{w=\left\{w_{n}\right\} \in \mathbb{X}: z_{n-1} \leq w_{n} \leq H z_{n-1}\right\},
$$

where $H>1$ is a constant. Let $\tau: \Omega \rightarrow \mathbb{X}$ be the map given by

$$
\begin{aligned}
& \tau(w)_{n}=z_{n-1} \quad \text { if } \tilde{n} \leq n \leq n_{0} \\
& \tau(w)_{n}=z_{n_{0}}+\sum_{i=n_{0}}^{n-1}\left(\frac{1}{a_{i}}\right)^{1 / \alpha}\left(\sum_{k=i}^{\infty} b_{k}\left(w_{k+q+1}\right)^{\alpha}\right)^{1 / \alpha} \quad \text { for } n \geq n_{0}+1 .
\end{aligned}
$$

Let $\tilde{n} \leq n \leq n_{0}$; clearly

$$
z_{n-1}=\tau(w)_{n} \leq H z_{n-1}
$$

Now let $n>n_{0}$. For $k \geq n_{0}+1$ it results that $k+q+1 \geq n_{0}+\bar{q}=\bar{n}+1=\tilde{n}$ and so for $w \in \Omega$ and $k \geq n_{0}+1$ :

$$
z_{k+q} \leq w_{k+q+1} \leq H z_{k+q} .
$$

Taking into account (5.14) and (5.28), we have

$$
\tau(w)_{n} \geq z_{n_{0}}+\sum_{i=n_{0}}^{n-1}\left(\frac{1}{a_{i}}\right)^{1 / \alpha}\left(\sum_{k=i}^{\infty} b_{k}\left(z_{k+q}\right)^{\alpha}\right)^{1 / \alpha}=z_{n}>z_{n-1} .
$$


Similarly, results for $n>n_{0}$ :

$$
\tau(w)_{n} \leq z_{n_{0}}+\sum_{i=n_{0}}^{n-1}\left(\frac{1}{a_{i}}\right)^{1 / \alpha}\left(\sum_{k=i}^{\infty} b_{k}\left(H z_{k+q}\right)^{\alpha}\right)^{1 / \alpha}=z_{n_{0}}+H z_{n}-H z_{n_{0}} .
$$

From (5.13) we obtain $z_{i-1} \geq z_{i}+h_{1}$ for $i \geq \bar{n}+1=\tilde{n}$ and so, since $n>\tilde{n}$, we have $z_{n-1} \geq z_{n}+h_{1}$. Consequently,

$$
\tau(w)_{n} \leq H z_{n-1}+z_{n_{0}}-H\left(h_{1}+z_{n_{0}}\right)
$$

Since $H>1$, we have $H\left(h_{1}+z_{n_{0}}\right) \geq z_{n_{0}}$ and so $\tau(w)_{n} \leq H z_{n-1}$. Thus $\tau(\Omega) \subset \Omega$. Since $\tau$ is continuous and compact in $\Omega$, by applying the Tychonov fixed point theorem, there exists $x$ such that $x=\tau(x)$. It is easy to verify that $x$ is a solution of (5.9) for $n$ large and, since $x \in \Omega$, we have $x \in \mathbb{M}_{\infty, 0}^{+}$, that is, the assertion.

Now we are able to prove Theorem 2.2.

Proof of Theorem 2.2. Claims $\left(i_{1}\right),\left(i_{2}\right)$ follow from Theorems 3.1, 3.2, and Proposition 4.1.

Claim $\left(i_{3}\right)$. From Theorem 3.2 $\left(i_{1}\right)$ and Proposition 4.1 we have $\mathbb{M}_{\ell, 0}^{+} \neq \varnothing, \mathbb{M}_{\infty, \ell}^{+}=\varnothing$. To prove $\mathbb{M}_{\infty, 0}^{+} \neq \varnothing$, let us show that $T_{\alpha}(1)=\infty$. Clearly, this is true if $q<1$, because $T_{\alpha}(q)=\infty$. If $q>1$, in view of (2.8) we have for large $n$

$$
\begin{aligned}
\sum_{k=0}^{n+q-1}\left(\frac{1}{a_{k}}\right)^{1 / \alpha} & =\sum_{k=0}^{n}\left(\frac{1}{a_{k}}\right)^{1 / \alpha}+\sum_{k=n+1}^{n+q-1}\left(\frac{1}{a_{k}}\right)^{1 / \alpha} \\
& \leq \sum_{k=0}^{n}\left(\frac{1}{a_{k}}\right)^{1 / \alpha}+h^{-1 / \alpha}(q-1)
\end{aligned}
$$

where $h$ is given by (5.12), and so $T_{\alpha}(1)=\infty$. In view of Theorem 2.1 $\left(i_{3}\right),(\mathrm{H})$ has intermediate solutions. Thus, Theorem 5.2 yields $\mathbb{M}_{\infty, 0}^{+} \neq \varnothing$ also for (1.1).

Claim $\left(i_{4}\right)$. Again from Theorem 3.2(i $\left.i_{1}\right)$ and Proposition 4.1, we have $\mathbb{M}_{\ell, 0}^{+}=\mathbb{M}_{\infty, \ell}^{+}=\varnothing$. Reasoning as in the proof of Claim $\left(i_{3}\right)$, we get $\mathbb{M}_{\infty, 0}^{+} \neq \varnothing$.

We close this section by an example illustrating the role of the delayed argument, when $a$ is rapidly varying at infinity.

Example 5.3. Consider (1.1) with $b_{n}=2^{-n^{2}+2 n}$ and

$$
a_{n+1}=2^{-n^{2}}\left(2^{(2 n+1) / \alpha}-1\right)^{-\alpha} \text { for } n \geq 0, a_{0}=1 \text {. }
$$

For any $\alpha>0$ we have

$$
\sum_{k=0}^{n}\left(\frac{1}{a_{k}}\right)^{1 / \alpha}=1+\sum_{k=1}^{n} \Delta 2^{(n-1)^{2} / \alpha}=2^{n^{2} / \alpha}
$$


and so $T_{\alpha}(1)=\infty$ and $T_{\alpha}(-1)<\infty$. Using the inequality $\sum_{i=n}^{\infty} 2^{-i^{2}+2 i} \geq 2^{-n^{2}+2 n}$, we obtain

$$
S_{\alpha} \geq \sum_{n=0}^{\infty}\left(\frac{1}{a_{n}}\right)^{1 / \alpha} 2^{\left(-n^{2}+2 n\right) / \alpha}=1+\sum_{n=1}^{\infty}\left(2^{2 n / \alpha}-2^{1 / \alpha}\right)=\infty .
$$

Thus, for $q \leq-1$ and any $\alpha,(1.1)$ satisfies the case $\left(C_{2}\right)$ and so, by Theorem 2.2 , it has intermediate and dominant solutions. Observe that if $q=1$, the case $\left(C_{4}\right)$ occurs, and, by Theorem 2.1, the half-linear equation $(\mathrm{H})$ is either oscillatory or all its solutions are intermediate. So, this example shows that if $q$ is negative, the case $\left(C_{2}\right)$ can occur for any $\alpha$.

Moreover, when $\alpha=1$ the linear equation

$$
\Delta\left(a_{n} \Delta x_{n}\right)+b_{n} x_{n+1}=0
$$

is oscillatory (see, e.g., [10, Remark 1.11.9]), and the corresponding delayed linear equation

$$
\Delta\left(a_{n} \Delta x_{n}\right)+b_{n} x_{n-\tau}=0, \quad \tau \in \mathbb{N},
$$

has, by Theorem 2.2, intermediate and dominant solutions. In comparison with Example 4.3, where the linear equation is nonoscillatory and the deviating argument may produce oscillatory solutions, in this case we have an opposite effect to the oscillation: the linear equation is oscillatory and the delay produces nonoscillatory solutions.

\section{Conclusion and open problems}

(1) The role of the deviating argument. By Theorems 2.1, 2.2, the existence of subdominant solutions does not depend on the deviating argument $q$, the one of dominant solutions can depend on $q$. Especially because $T_{\alpha}(1-\tau) \leq T_{\alpha}(1) \leq T_{\alpha}(1+\tau)$, we obtain a possible discrepancy concerning unbounded solutions of $(\mathrm{H})$ and an equation with deviating argument. For instance, if the case $\left(C_{1}\right)$ holds, $(\mathrm{H})$ has dominant solutions and no intermediate solutions, while it may occur that $(\mathrm{H}+)$ has intermediate solutions and no dominant solutions. Similarly, the delayed argument may cause the opposite phenomena: if the case $\left(C_{3}\right)$ holds, $(H)$ has intermediate solutions and no dominant solutions, while it may occur that $(\mathrm{H}-)$ has dominant solutions and no intermediate solutions. Clearly, the deviating argument can produce also the oscillation, or nonoscillation, as Examples 4.3 and 5.3 show.

(2) Open problems. As we noticed in Section 4, the nonoscillation of all solutions of (1.1) with $q \neq 1$ is an open problem.

For this reason, when $T_{\alpha}(q)=\infty(q \neq 1)$, the existence of intermediate solutions has been obtained by means of a comparison result between $(\mathrm{H})$ and (1.1), say Theorem 5.2. Such a result shows that the deviating argument does not have any influence on the existence of intermediate solutions, provided (2.8) holds. Especially, if the classical half-linear equation $(\mathrm{H})$ has intermediate solutions and $(2.8)$ holds, then also $(\mathrm{H}+)$ and $(\mathrm{H}-)$ have intermediate solutions for any $\tau \in \mathbb{Z}, \tau \geq 1$.

If (2.8) is not satisfied, Theorem 5.2 can fail, as Example 5.1 illustrates. Is condition (2.8) necessary for the validity of this theorem and, consequently, of Theorem 2.2? By means of an argument similar to the one given in the proof of Theorem $2.2\left(i_{3}\right)$ we have that $(2.8)$ yields that the series $T_{\alpha}(p), T_{\alpha}(q)$ have the same character, that is, are both convergent or divergent for 
any $p, q \in \mathbb{Z}$. Now, the following question arises: is the statement of these theorems valid by assuming, instead of (2.8), that $T_{\alpha}(p), T_{\alpha}(q)$ are both convergent or divergent?

(3) Future investigations. The future studies will be addressed to extend the results of this paper, especially those concerning the intermediate solutions, to Emden-Fowler-type equations of the form

$$
\Delta\left(a_{n}\left|\Delta x_{n}\right|^{\alpha} \operatorname{sgn} \Delta x_{n}\right)+b_{n}\left|x_{n+q}\right|^{\beta} \operatorname{sgn} x_{n+q}=0, \quad \alpha \neq \beta .
$$

\section{Acknowledgment}

Supported by the Research Project 0021622409 of the Ministery of Education of the Czech Republic, and Grant no. 201/07/0145 of the Czech Grant Agency.

\section{References}

[1] M. Cecchi, Z. Došlá, and M. Marini, "Nonoscillatory half-linear difference equations and recessive solutions," Advances in Difference Equations, vol. 2005, no. 2, pp. 193-204, 2005.

[2] M. Cecchi, Z. Došlá, M. Marini, and I. Vrkoč, "Summation inequalities and half-linear difference equations," Journal of Mathematical Analysis and Applications, vol. 302, no. 1, pp. 1-13, 2005.

[3] M. Cecchi, Z. Došlá, and M. Marini, "Intermediate solutions for nonlinear difference equations with $p$-Laplacian," in Proceedings of the 11th International Conference on Difference Equations and Applications (ICDEA '06), S. Elaydi, K. Nishimura, and M. Shishikura, Eds., Advanced Studies in Pure Mathematics, Kyoto, Japan, July 2006.

[4] H. A. El-Morshedy and S. R. Grace, "Comparison theorems for second order nonlinear difference equations," Journal of Mathematical Analysis and Applications, vol. 306, no. 1, pp. 106-121, 2005.

[5] H.-F. Huo and W.-T. Li, "Oscillation of certain two-dimensional nonlinear difference systems," Computers \& Mathematics with Applications, vol. 45, no. 6-9, pp. 1221-1226, 2003.

[6] W.-T. Li, "Classification schemes for nonoscillatory solutions of two-dimensional nonlinear difference systems," Computers and Mathematics with Applications, vol. 42, no. 3-5, pp. 341-355, 2001.

[7] P. ̌̌ehák, "Oscillatory properties of second order half-linear difference equations," Czechoslovak Mathematical Journal, vol. 51, no. 2, pp. 303-321, 2001.

[8] S. H. Saker, "Oscillation of second-order perturbed nonlinear difference equations," Applied Mathematics and Computation, vol. 144, no. 2-3, pp. 305-324, 2003.

[9] G. Zhang, S. S. Cheng, and Y. Gao, "Classification schemes for positive solutions of a second-order nonlinear difference equation," Journal of Computational and Applied Mathematics, vol. 101, no. 1-2, pp. 39-51, 1999.

[10] R. P. Agarwal, M. Bohner, S. R. Grace, and D. O'Regan, Discrete Oscillation Theory, Hindawi Publishing Corporation, New York, NY, USA, 2005.

[11] O. Došlý and P. Řehák, Half-Linear Differential Equations, vol. 202 of North-Holland Mathematics Studies, Elsevier Science B.V., Amsterdam, The Netherlands, 2005.

[12] R. P. Agarwal, S. R. Grace, and D. O'Regan, Oscillation Theory for Second Order Linear, HalfLinear, Superlinear and Sublinear Dynamic Equations, Kluwer Academic Publishers, Dordrecht, The Netherlands, 2002.

[13] H. Hoshino, R. Imabayashi, T. Kusano, and T. Tanigawa, "On second-order half-linear oscillations," Advances in Mathematical Sciences and Applications, vol. 8, no. 1, pp. 199-216, 1998.

[14] M. Cecchi, Z. Došlá, and M. Marini, "On intermediate solutions and the Wronskian for half-linear differential equations," Journal of Mathematical Analysis and Applications, vol. 336, no. 2, pp. 905-918, 2007.

[15] J. Wang, “Oscillation and nonoscillation theorems for a class of second order quasilinear functionaldifferential equations," Hiroshima Mathematical Journal, vol. 27, no. 3, pp. 449-466, 1997. 